\title{
Nonlinear Electric Field Effects at a Continuous Mott-Hubbard Transition
}

\author{
A. Husmann, ${ }^{1}$ J. Brooke, ${ }^{2}$ T. F. Rosenbaum, ${ }^{2}$ X. Yao, ${ }^{3}$ and J. M. Honig ${ }^{3}$ \\ ${ }^{1}$ Clarendon Laboratory, University of Oxford, Oxford OXI 3PU, England \\ ${ }^{2}$ The James Franck Institute and Department of Physics, The University of Chicago, Chicago, Illinois 60637 \\ ${ }^{3}$ Department of Chemistry, Purdue University, West Lafayette, Indiana 47907
}

(Received 27 September 1999)

\begin{abstract}
We characterize the non-Ohmic portion of the conductivity at temperatures $T<1 \mathrm{~K}$ in the highly correlated transition metal chalcogenide $\mathrm{Ni}(\mathrm{S}, \mathrm{Se})_{2}$. Pressure tuning of the $T=0$ metal-insulator transition reveals the influence of the quantum critical point and permits a direct determination of the dynamical critical exponent $z=2.7_{-0.4}^{+0.3}$. Within the framework of finite temperature scaling, we find that the spatial correlation length exponent $\nu$ and the conductivity exponent $\mu$ differ.
\end{abstract}

PACS numbers: $71.30 .+\mathrm{h}, 71.27 .+\mathrm{a}, 72.80 . \mathrm{Ga}$

Zero temperature phase transitions are fundamentally different from their finite temperature counterparts. Quantum fluctuations on a time scale set by Planck's constant and the characteristic energy of the system inextricably link the static and dynamic response. In the lexicon of continuous phase transitions, the static critical exponent which describes a thermodynamic property of a classical system must be joined in the quantum limit by a new dynamical exponent.

The metal-insulator transition at zero temperature represents a particularly difficult limit of the problem. The $T=0$ electrical conductivity, $\sigma(0)$, changes from zero in the insulator to a finite value in the metal, but it does not qualify as a thermodynamic quantity. Nonetheless, scaling arguments appear to be persuasive in the single electron limit $[1,2]$. Strong electron-electron interactions cloud the theoretical picture [3]. Fundamental questions - the role played by external electric and magnetic fields, the values of the static and dynamical exponents, the number of universality classes - remain unresolved.

The experimental situation for highly correlated materials is complicated by the usual but unfortunate coincidence between electronic transitions and structural changes that shroud the critical behavior. An exception to this rule is the transition-metal chalcogenide $\mathrm{NiS}_{2-x} \mathrm{Se}_{x}$, a Mott-Hubbard system [4] without a symmetry change at an effectively continuous $T=0$ metal-insulator transition [5]. Both changing stoichiometry $x$ and applying hydrostatic pressure $P$ can tune the transition [6]; the pertinent $P-T$ phase diagram for $x=0.44$ is shown in Fig. 1 [7]. Both Se substitution and applied pressure increase the bandwidth without moving the system away from half filling.

Pressure tuning of the transition yields a critical form $\sigma(0) \sim\left[\left(P-P_{c}\right) / P_{c}\right]^{\mu}$ with the conductivity exponent $\mu=1.1 \pm 0.2$, while temperature scans for $T<0.8 \mathrm{~K}$ very close to $P_{c}$ find a critical dynamical response $[\sigma-$ $\sigma(0)] \sim T^{0.22 \pm 0.02}$ [5]. Within the finite-size scaling picture of quantum phase transitions [8], these results give $z \nu=4.6 \pm 0.4$, where $\nu$ characterizes the divergence of the spatial correlation length and the dynamical critical exponent $z$ relates the temporal and spatial dimensions. In the case of noninteracting electrons, Wegner scaling [1] asserts that $\mu=(d-2) \nu=\nu$ for three dimensions $d$. With interactions present, it is not possible to deconvolute $z$ and $\nu$ without additional experimental probes of the system.

In this Letter, we characterize the nonlinear $I-V$ response of barely metallic $\mathrm{NiS}_{1.56} \mathrm{Se}_{0.44}$ at temperatures below $1 \mathrm{~K}$ as $\left(P-P_{c}\right) \rightarrow 0$. With pressure as a tuning parameter, we are able to assess the impact of the MottHubbard quantum critical point on the non-Ohmic conductivity. Moreover, from $\sigma(T, E)$, where $E$ is the electric field, we can determine $\nu$ and $z$ independently, and we find that $\mu \neq \nu$. The prevalent assumption that measuring the critical behavior of $\sigma(0)$ reveals the divergent nature

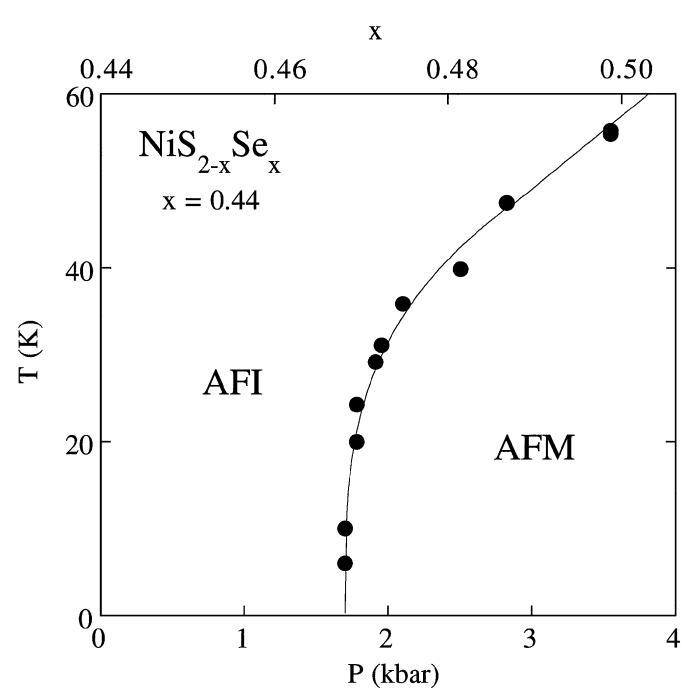

FIG. 1. Pressure-temperature phase diagram for crystals of $\mathrm{NiS}_{1.56} \mathrm{Se}_{0.44}$. The critical pressure for the $T=0$ metalinsulator transition is less than 2 kbar. Each kbar of external pressure corresponds to a chemical pressure $\Delta x=0.017$. $\mathrm{AFI}=$ antiferromagnetic insulator; $\mathrm{AFM}=$ antiferromagnetic metal. 
of the correlation (localization) length does not hold for this highly correlated transition.

Single crystals of $\mathrm{NiS}_{2-x} \mathrm{Se}_{x}$ were grown by a new tellurium flux method, which minimizes excess impurity concentrations [9]. The amount of Se substitution $x$ is determined from $x$-ray diffraction measurements of the lattice parameter using Vegard's law. Ion microprobe studies indicate an actual stoichiometry $\mathrm{Ni}(\mathrm{S}, \mathrm{Se})_{2-\delta}$, with $\delta=0.03 \pm 0.01$ across the breadth of a crystal. The worry that chalcogenide vacancies could provide a parallel surface conduction path was explicitly ruled out in our measurements of the metal by comparing results on single crystals with different aspect ratios. We performed nonlinear $I-V$ measurements using square-wave excitation from a frequency synthesizer detected by a digital lock-in amplifier. The square wave permits monotonic variation in the electric field without unnecessary rounding of the threshold response, while retaining the increased sensitivity of a finite frequency technique. The nanowatt power levels at threshold serve to obviate concerns about $I^{2} R$ lattice heating. The errors in the relative values of $\sigma$ and $E$ are less than $0.01 \%$, but there is an absolute uncertainty of $25 \%$ because of the small size of the crystals $\left(\sim 0.5 \times 0.5 \times 3 \mathrm{~mm}^{3}\right)$ and the spread of the silver paint electrical contacts. The $\mathrm{BeCu}$ piston-anvil self-locking pressure clamp, with silicone oil as the pressure medium and a chip of $\left(\mathrm{V}_{0.99} \mathrm{Ti}_{0.01}\right)_{2} \mathrm{O}_{3}$ as a manometer [10], was cycled between $T=0.3$ and $6 \mathrm{~K}$ in a charcoal-pumped ${ }^{3} \mathrm{He}$ cryostat.

We plot in Fig. 2 the raw data: $\sigma(E)$ for $0.35 \leq T \leq$ $0.8 \mathrm{~K}$ at three hydrostatic pressures $P$. The critical pressure for the metal-insulator transition is $P_{c}=1.51 \mathrm{kbar}$. For $E<10^{-4} \mathrm{~V} / \mathrm{cm}$ the response is Ohmic [11]. At higher excitations the sample becomes more conducting. The size of the non-Ohmic contribution $\Delta \sigma / \sigma$ rises rapidly as the metal-insulator transition is approached from above, tracking the change in $\sigma$. The dimensionless change in the conductivity grows from $1 \%$ at $\left(P-P_{c}\right) / P_{c}=0.13$ to $10 \%$ at $\left(P-P_{c}\right) / P_{c}=0.02$ over 1.5 decades in $E$. We note that if the chemical pressure from the Se substitution is included in $P_{c}$, then the dimensionless distance to the critical point would be a factor of 20 smaller (see Fig. 1).

At each pressure, the threshold electric field $E_{\mathrm{th}}$ for the nonlinear $I-V$ response moves to higher $E$ with increasing $T$. In the context of finite-size scaling about a quantum critical point, this trend can be understood as a crossover from scaling controlled by temperature [5] to scaling controlled by the electric field. It is qualitatively consistent as well with $I R$ heating of the electrons. In fact, in the noninteracting case (e.g., the localization problem) the electric field is not a scaling variable, and non-Ohmic measurements of low density $\mathrm{Si}$ MOSFETs [12] have been described both within the scaling paradigm and as heating of the two-dimensional electron gas [13].

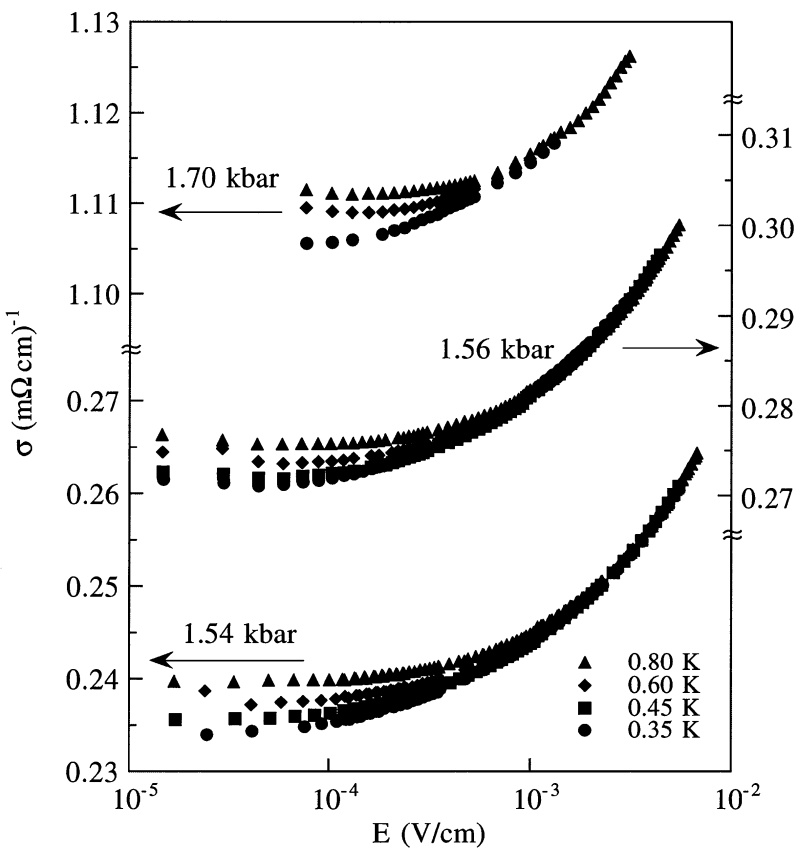

FIG. 2. Variation of the conductivity $\sigma$ with electric field $E$ at temperatures $0.35 \leq T \leq 0.80 \mathrm{~K}$ for a series of pressures just above $P_{c}=1.51 \mathrm{kbar}$. The nonlinear response becomes apparent above a threshold field $E_{\mathrm{th}} \sim 10^{-4} \mathrm{~V} / \mathrm{cm}$.

By comparing the threshold response at different pressures, we find that simple IR heating of the electron gas is not compatible with our data. We plot in Fig. 3 the normalized change in conductivity at $T=0.35 \mathrm{~K}$ for $P=1.54$,

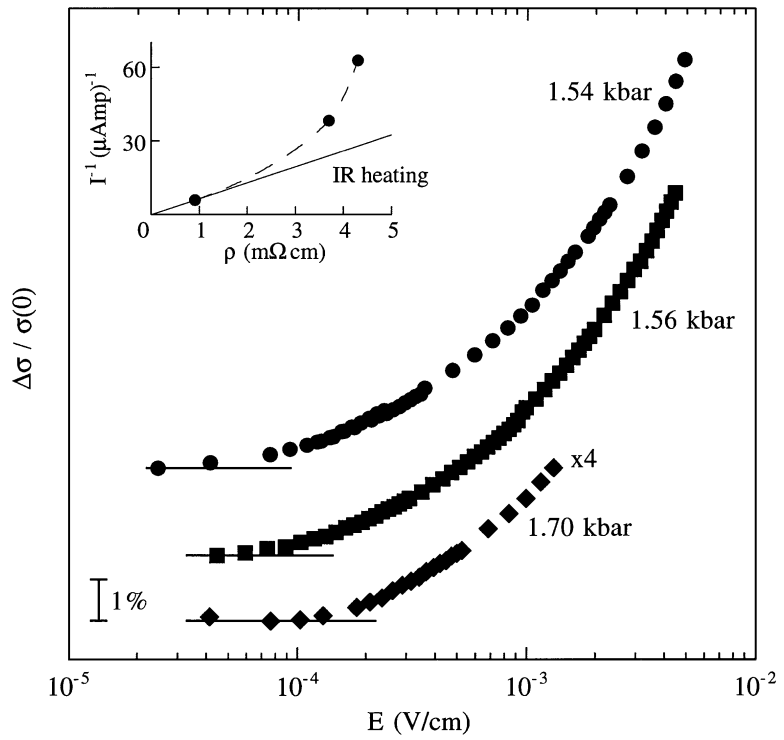

FIG. 3. The threshold field moves to larger $E$ with increasing $P$. Simple $I R$ heating of the electron gas would give $E_{\text {th }}$ independent of $P$. All data are taken at $T=0.35 \mathrm{~K}$ and the vertical scale in units of normalized change in $\sigma$ is multiplied by 4 at $P=1.70$ kbar. Inset: Rapid increase of the inverse threshold current $I$ with sample resistivity $\rho$ as the metal-insulator transition is approached. 
1.56, and 1.70 kbar. Electron heating implies a constant threshold for non-Ohmicity, while $E_{\text {th }}$ clearly moves down as the transition at $P=1.51 \mathrm{kbar}$ is approached from above [14]. We highlight in the inset the importance of the Mott-Hubbard critical point: the inverse of the threshold current $I$ grows rapidly as the sample resistivity increases near the transition. Here we have chosen $E_{\mathrm{th}}$ as the linear extrapolation from the beginning of the rise in $\Delta \sigma / \sigma$ back to the baseline, corresponding to $\sim 5 \%$ of the total change in $\Delta \sigma / \sigma$, but any such criterion will provide the same qualitative result.

Strong electron-electron interactions, as are present for $\mathrm{Ni}(\mathrm{S}, \mathrm{Se})_{2}$, appear to make the electric field a pertinent scaling variable at the metal-insulator transition. While the dynamical response is described by $T^{1 / \nu z}\left(\omega^{1 / \nu z}\right)$, the electric field involves an additional length scale. Hence, the conductivity should vary as $E^{1 / \nu(z+1)}$ in the quantum critical regime. Converting $\sigma\left(E>E_{\text {th }}\right)$ to an effective temperature $T_{\text {eff }}$ by knowing $\sigma(T, E \rightarrow 0)$ at the same $P$, permits a direct measurement of the dynamical exponent $z$ [15]. The static exponent $\nu$ cancels in the conversion, leaving $T_{\text {eff }} \sim E^{z /(z+1)}$ in the regime where the scaling is controlled by the electric field length.

We plot in Fig. 4 the effective temperature as a function of $E$ for $\left(P-P_{c}\right) / P_{c}=0.03$. The data for different real $T$ overlay at large field, as expected, and demonstrate reasonable power law behavior. Extension to higher $T_{\text {eff }}$ is precluded by concerns over potential lattice heating. The best fit slope over one decade in $E$ gives $z /(z+1)=0.73_{-0.06}^{+0.03}$. Analogous treatments at $\left(P-P_{c}\right) / P_{c}=0.02$ and 0.13 give $z /(z+1)=$ $0.72_{-0.06}^{+0.02}$ and $0.73_{-0.06}^{+0.03}$, respectively. The dominant source of systematic error is the sensitivity to the range of

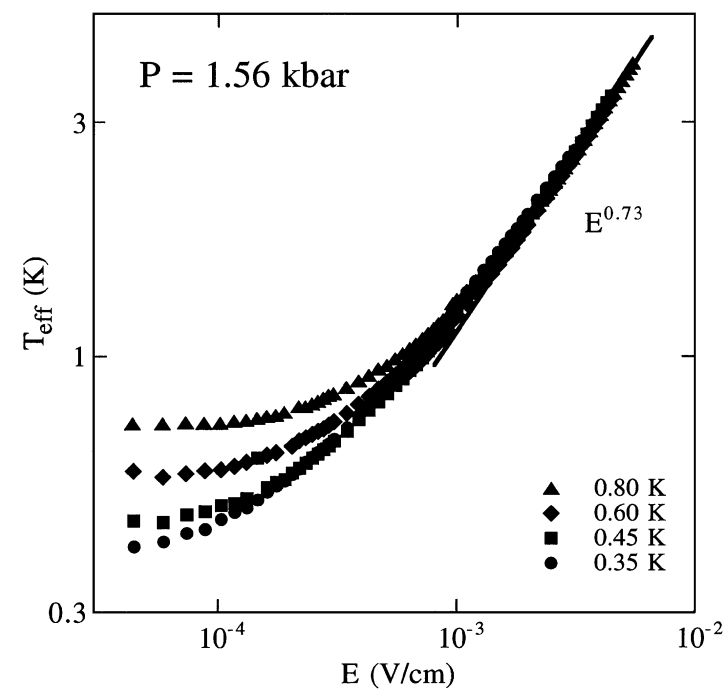

FIG. 4. Effective electron temperature $T_{\text {eff }}$ vs electric field $E$ at $\left(P-P_{c}\right) / P_{c}=0.03$. The data for $0.35 \leq T \leq 0.80 \mathrm{~K}$ converge in the scaling regime at large $E$. The slope on this log-log plot is $z /(z+1)$, where $z$ is the dynamical critical exponent. The solid line over one decade in $E$ is the best fit slope $0.73_{-0.06}^{+0.03}$. fit. Combining the results at all three pressures yields a best fit value $z /(z+1)=0.73_{-0.03}^{+0.02}$, so that $z=2.7_{-0.4}^{+0.3}$.

If one assumes that the conductivity and spatial correlation length exponents are identical, $\mu=\nu \sim 1$, then the previously ascertained dynamical response for $\mathrm{NiS}_{2-x} \mathrm{Se}_{x}$ [5] would constrain $z$ to lie between 4 and 5. The present analysis of electric field scaling at the pressure-tuned metal-insulator transition establishes directly that the dynamical critical exponent $z$ has a value between 2 and 3 , with a best fit value more than 4 standard deviations below $z=4$. Combining the results from temperature and electric field excursions about the Mott-Hubbard critical point, $z \nu=4.6 \pm 0.4$ and $z=2.7_{-0.4}^{+0.3}$, yields a static critical exponent $\nu=1.7_{-0.2}^{+0.3}$. Dynamical scaling of the full body of data, $\sigma(T, P, E)$, is consistent with these values of the static and dynamical critical exponents, but with negligible overlap between data sets at different pressures.

We are now able to address the questions posed in the introduction about the $T=0$ metal-insulator transition with strong correlations: the role played by an external electric field, the values of the static and dynamical exponents, the number of universality classes [16]. As demonstrated in Figs. 2 and 3, the evolution with $P$ of the non-Ohmic contribution to $\sigma(E)$ reflects the presence of the quantum critical point. The Mott-Hubbard transition and the pure localization transition differ on this account. Conversion to $T_{\text {eff }}$ (Fig. 4) permits an independent fix on $z$, with the corollary that Wegner scaling $(\mu=\nu=1)$, derived for noninteracting electrons, is violated here. The large effective dimensionality of the $\mathrm{NiS}_{2-x} \mathrm{Se}_{x}$ system, $(d+z)>5$, makes violations of hyperscaling less surprising [17], but does indicate that measuring the conductivity exponent alone at any continuous metal-insulator transition will not suffice to peg $\nu$ with confidence. Unresolved is the issue of universality: Are $z=2.7_{-0.4}^{+0.3}$ and $\nu=1.7_{-0.2}^{+0.3}$ common values for all $T=0$ Mott-Hubbard transitions, or specific to the compound at hand? Effectively continuous and practicably tuned metal-insulator transitions in other highly correlated materials must still be identified to proceed on this line of inquiry.

We are grateful to C. Roeser for expert technical help and to R. N. Bhatt and S. M. Girvin for illuminating discussions. T. F. R. thanks the Aspen Center for Physics where some of this work was performed. The work at the University of Chicago was supported by NSF No. DMR9801824; that at Purdue was supported by NSF No. DMR9612130.

[1] F. J. Wegner, Z. Phys. B 25, 327 (1976).

[2] E. Abrahams, P. W. Anderson, D. C. Licciardello, and T. V. Ramakrishnan, Phys. Rev. Lett. 42, 673 (1979).

[3] For a recent review, see D. Belitz and T. R. Kirkpatrick, Rev. Mod. Phys. 66, 261 (1994).

[4] Although $\mathrm{NiS}_{2-x} \mathrm{Se}_{x}$ has charge transfer characteristics, it is commonly analyzed within a Mott-Hubbard framework. 
See, for example, J. Zaanen, G. A. Sawatzky, and J. W. Allen, Phys. Rev. Lett. 55, 418 (1985); A. Y. Matsuura et al., Phys. Rev. B 53, R7584 (1996); J. M. Honig and J. Spalek, Chem. Mater. 10, 2910 (1998).

[5] A. Husmann, D. S. Jin, Y. V. Zastavker, T. F. Rosenbaum, X. Yao, and J. M. Honig, Science 274, 1874 (1996).

[6] J. A. Wilson, in The Metallic and Nonmetallic States of Matter, edited by P. P. Edwards and C. N. R. Rao (Taylor and Francis, London, 1985), pp. 215-260.

[7] We have characterized two $\mathrm{NiS}_{1.56} \mathrm{Se}_{0.44}$ crystals with critical pressures for the insulator-metal transition of $P_{c}=$ 1.51 and $1.67 \mathrm{kbar}$, respectively. High temperature data (Fig. 1) were collected only for the $P_{c}=1.67$ kbar crystal. These values of $P_{c}$ correspond to the expected $x_{c}=0.47$; the difference of $0.16 \mathrm{kbar}$ corresponds to a difference in $x \sim 0.003$.

[8] S. L. Sondhi, S. M. Girvin, J. P. Carini, and D. Shahar, Rev. Mod. Phys. 69, 315 (1997).

[9] X. Yao and J. M. Honig, Mater. Res. Bull. 29, 709 (1994); X. Yao, J. M. Honig, T. Hogan, C. Kannewurf, and J. Spalek, Phys. Rev. B 54, 17469 (1996).
[10] T.F. Rosenbaum, S. A. Carter, and J. M. Honig, Rev. Sci. Instrum. 67, 617 (1996).

[11] The slight increase in conductivity at the smallest fields is an artifact related to phase shifts in the detection circuitry.

[12] S. V. Kravchenko, D. Simonian, M. P. Sarachik, W. Mason, and J. E. Furneaux, Phys. Rev. Lett. 77, 4938 (1996).

[13] B. L. Altshuler and D. L. Maslov, Phys. Rev. Lett. 82, 145 (1999).

[14] The contact resistance remains constant for these pressures so $I^{2} R$ lattice heating also is incompatible with the data. The relevance of a phonon bottleneck effect at $\mathrm{mK}$ temperatures is harder to assess, although it seems less likely for 3D systems than for 2D systems. See E. Chow, H. P. Wei, S. M. Girvin, and M. Shayegan, Phys. Rev. Lett. 77, 1143 (1996).

[15] H. P. Wei, L. W. Engel, and D. C. Tsui, Phys. Rev. B 50, 14609 (1994).

[16] G. Moeller, Q. Si, G. Kotliar, M. Rozenberg, and D. S. Fisher, Phys. Rev. Lett. 74, 2082 (1995).

[17] T. R. Kirkpatrick and D. Belitz, Phys. Rev. Lett. 74, 1178 (1995). 